\title{
On the Processes of Heat Transfer in Earth's Mantle
}

\author{
Lubimova H. A. $\left({ }^{*}\right)$ \\ (Institute of Earth's Physies, Academy of Sciences, USSR)
}

Ricevuto il 7 dicembre 1960

The attraction of the data of the physics of semiconductors and dielectrics created new possibilities of studying the thermal regime and the thermal history of the Earth. For instance the role of the radiative thermal conductivity was investigated (Clark 1956, Preston 1956) which in addition to the lattice thermal conductivity must lead to an intensive heat transfer in the deep interior of the Earth.

The investigation of the pattern of the lattice part of the thermal ronductivity drew to a conclusion about the presence of the summary thermal conductivity minimum in the 50 to $100 \mathrm{~km}$ of the mantle. This is of a decisive importance for the study of the Earth's thermal regime. The low thermal conductivity of the Earth's upper layers hinders an intensive loss of heat, provides for the accumulation of heat and heating of the deep interior of the Earth but not its cooling. Due to the minimum of the thermal conductivity the temperature gradient has an increased value which is sufficient to explain the observed lowering of the velocities of seismic waves at these depths (Lubimova H. A. 1958, 1959).

The new data about the properties of ionic semi-conductors and non-metallic materials in the range of high temperatures indicate the existence of one more process - energy transfer by the excited states of atoms - excitons. The possibility of the existence of exciton states in a given material is established by the absorption spectra of the material.

Combinations $\mathrm{MgO}$ and $\mathrm{Mg}_{2} \mathrm{SiO}_{4}$ are the main components of the typical earth rocks and meteorites. Kingery (1954) measured the thermal conductivity of these materials at high temperatures. Fig. 1 shows that at $T>1000^{\circ} \mathrm{C}$ the thermal conductivity passes through its mi-

(*) Paper read at the Assembly of the I.U.G.G., 1960. 
nirnum and begins to grow with the increase of $T$. The deviation from the lattice thermal conductivity law $\frac{1}{\overline{T^{\prime}}}$ or $\frac{1}{T^{5 / 4}}$ increases with the increase of temperature (Fig. 1b). Let us compare the data on the thermal conductivity with the data concerning the absorption spectra of these materials.

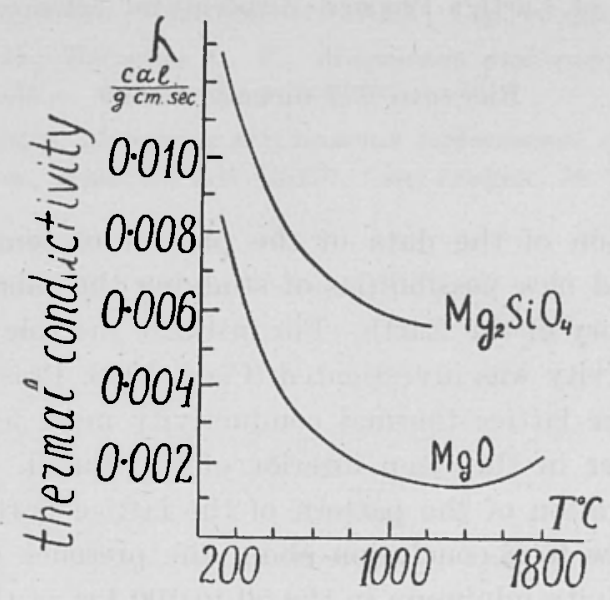

Fig. 1. - The thermal conductivity variation for $\mathrm{Mg}_{2} \mathrm{SiO}_{4}$ and $\mathrm{MgO}$ with temperature.

The absorption spectrum of olivine was studied by Clark (1957), special attention being paid to the existence of the transparancy interval providing for the radiative energy transfer. The presence of several absorption peaks in the transparancy interval remained unexplained although a hypothesis about their possible exciton origin was assumed.

Reiling and Hensley (1958) found strong and weak absorption peaks in the spectrum $\mathrm{MgO}$ at $T=-170^{\circ} ;+25^{\circ}$ and $+150^{\circ}$ and ascribed them to solely exciton state. Exciton peaks are found in the spectra of a number of other ion and also atom combinations, such as $\mathrm{KI}, \mathrm{KBr}$, $\mathrm{InSb}, \mathrm{Cu}_{2} \mathrm{O}, \mathrm{PbI}_{2}$, TeBr, Ge and others (Haken, 1959). In atomic semiconductors these lines can be observed only at low temperatures when the background absorption is weakened. Whereas in ion crystals the exciton absorption is so strong that it can be observed even at high temperatures (Martienssen, 1959). Clark's observations are made at room temperature. 
The distinguishing of exciton lines is facilitated by the fact that a definite type of excitons - non-localized excitons give narrow lines in their spectra with the frequences satisfying a hydrogen-like law:

$$
v_{n}=v_{\infty}-\frac{R_{e:}}{n^{2}} \text {. }
$$

We found that several narrow lines in the absorption spectrum of olivine can be identified with the exciton state of a definite quant num-

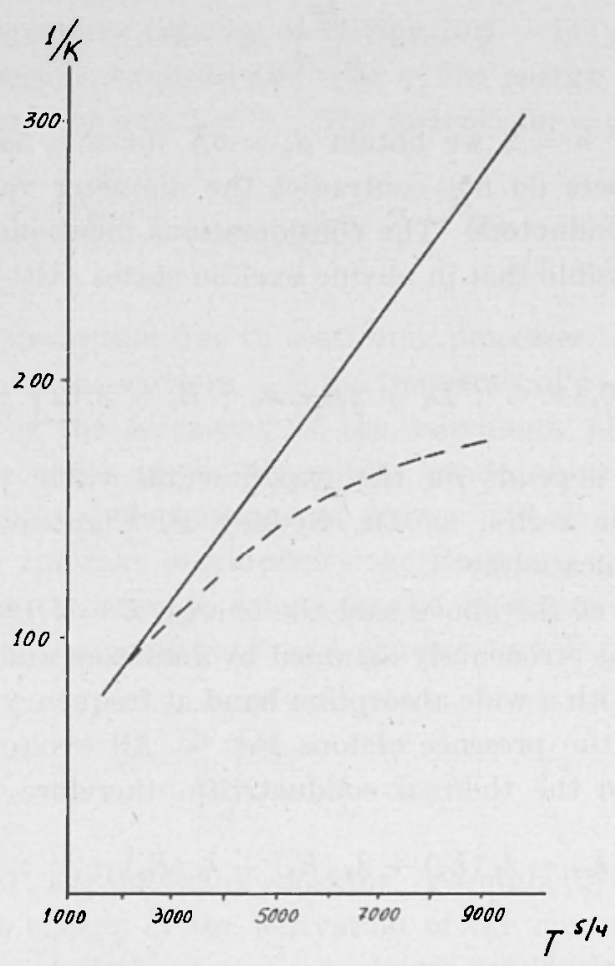

Fig. 1b. - The deviation of the thermal conductivity of $\mathrm{Mg}_{2} \mathrm{SiO}_{4}$ from the lattice conductivity low $\frac{1}{T^{5 / 4}}$ with temperature.

ber $n=1,2,3$ if to assume that $v_{\infty}=29000 \mathrm{~cm}^{-1}, R_{e x}=25800 \mathrm{~cm}^{-1}$. These are lines with frequences $3200,22600,25200,29100 \mathrm{~cm}^{-1}$. The condensation of the lines occurs in the direction of the ultra-violet absorption, the interface frequency of the series coincides with the edge frequency of this band equal to $29100 \mathrm{~cm}^{-1}$. 
The value of the constant $R_{e x}=25800 \mathrm{~cm}^{-1}$ yields a correct value for the reduced mass of exciton

$$
\mu_{e x}^{*}=\frac{R_{e x} \cdot n_{o}{ }^{4} \cdot m}{K} \sim 2 m
$$

where $R=1,097 \cdot 10^{5} \mathrm{~cm}^{-1}$ is the Ridberg's constant in wave numbers, $m=9,11 \cdot 10^{-28} \mathrm{~g}$ is the electron mass, $n_{0}$ - is the refractive index. The diameters of excitons can be calculated:

$$
a_{e}=2 \frac{h^{2}}{\mu^{*} e^{-}} n_{o}^{2} \cdot n^{2}
$$

For instance for $n=2$ we obtain $d_{2}=6 \AA$ for $n=5 d_{5}=150 \AA$.

These numbers do not contradict the diameter values established for other semi-conductors. The considerations mentioned above permit to believe it plausible that in olivine exciton states exist with the energy of excitation:

$$
E_{1}=0,4 \text { e.v. ; } E_{2}=2,8 \text { e.v. } ; E_{3}=3,12 \text { e. } v . \ldots
$$

This result depends on the experimental value of the interface frequency of the series, as Dr. Sydney P. Clark correctly marked (private communication).

In the light of the above said the energy $E=1,19$ has no relation to excitons as was erroneously assumed by Jamieson and Lawson (1958). It is associated with a wide absorption band at frequency $v=10000 \mathrm{~cm}^{-1}$ which is due to the presence of ions $\mathrm{Fe}^{++}$. All excitons should make a contribution to the thermal conductivity, therefore

$$
\lambda_{e x}=\lambda_{1}\left(E_{1}\right)+\lambda_{2}\left(E_{2}\right)+\lambda_{3}\left(E_{3}\right)+\ldots
$$

where

$$
\lambda_{i}=\frac{16 \pi}{3}\left(\frac{k}{h}\right)^{3} \mu^{*} \cdot T^{2} \cdot l \cdot e^{-E_{i} / K T} \cdot\left[\left(\frac{E_{i}}{K T}+2\right)^{2}+2\right]
$$

$l=l_{o} / T$ being the length of the free run of excitons. In normal condi-

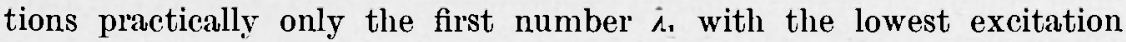
energy plays the main role. With the growth of temperature exciton peaks shift in the direction of longer waves and lower energies (Martienssen, 1959). Therefore at high temperatures the contribution from the subsequent terms must increase and the role of the exciton thermal conductivity must increase. The rate of the peaks' movements with 
the growth of $T$ in the direction of greater energies in $\mathrm{MgO}$ is $-5.10^{-4}$ e.v. ${ }^{\circ} K$ (Reiling and Hensley 1958).

The contrary effect on the parameters of the radiative thermal conductivity $\lambda_{r}=16 \sigma n_{o}^{3} T^{3} / 3 \varepsilon$ is caused by temperature. The absorption coefficient $\varepsilon$ of the radiative energy depends on $T$ and $\nu$ in a complicated way. At different frequencies different absorption mechanisms exist but they all lead to an increase of absorption with the growth of temperature and pressure and the dying away of the radiative heat transfer at great depth.

In the transparancy interval of olivine $(0.3-11) \mu$ the absorption is due to free carriers, excitons and split of the energy levels of the unfilled electron shells of ions $\mathrm{Fe}^{++}$. The formula for $\varepsilon$ can be written as $\varepsilon=\varepsilon_{o}+\varepsilon_{1} \frac{\exp \left(-\frac{E_{\mathrm{g}}}{K T}\right)}{v^{2}}+\sum \varepsilon^{i} \exp \left(-a_{i} \frac{h\left(\nu_{i}-\nu\right)}{K T}\right)+\varepsilon_{3} \frac{\nu_{\max }^{3}}{\Delta \nu}$

where $\varepsilon_{o}$ is the absorption due to scattering processes. $E_{g}$ is the energy of the activation of the carriers, $\nu_{\imath}$ is the frequency of $i$ - exciton absorption peak, $\nu_{\max }$ is the frequency of the maximum absorption by ion $\mathrm{Fe}^{++}, \Delta v$ is the width of the absorption band due to $\mathrm{Fe}^{++}$.

Such complicated dependence of $\varepsilon$ on $\nu$ and $T$ is rather difficult to be used. For the sake of simplicity the Rosseland averaging formula can be used, then the average value $\bar{\varepsilon}$ for the spectrum must be calculated for the given temperature $T$ and the pressure $P$ from the formula

$$
\frac{1}{\varepsilon}=\frac{15}{4 \pi^{4}} \int_{0}^{\infty} \frac{1}{\varepsilon_{u}} \cdot \frac{u^{*} e^{-u}}{\left(1-e^{-u}\right)^{2}} \cdot d u
$$

where $u=h v / K T, h$ is the Plank constant. Formula [2] for $\varepsilon$ includes the value $E_{g}$ - the energy of the activation of the current carriers. The energy of the activation of the ion electro-conductivity predominant in the upper layers of the Earth is studied in a number of papers (see review of $D$. C. Tozer, 1959). The dependence of the activation energy of the intrinsic electron conductivity predominant in the lower layers of the mantle on $T$ and $P$ we write as

$$
E_{\boldsymbol{g}}-E_{\boldsymbol{g}}+\boldsymbol{r} \frac{\partial E_{g}}{\partial p}-\bar{u} \boldsymbol{M} \boldsymbol{T} \frac{\partial E_{g}}{\partial p} \quad \rho T
$$

where $\alpha$ is the coefficient of the thermal expansion; $K$ - is the incompressibility, $E_{g}^{o}$ is the width of the energy gap at normal pressure and 
temperature. The second term allows for the effect of the pressure on the width of the zone. According to the data of Balchan A. S. and Drickamer H. G. (1959) $\frac{\partial E_{g}}{\partial p}=-2,5 \cdot 10^{-6} \frac{e . v .}{d y n e \cdot \mathrm{cm}^{2}}$. The third term allows for the effect of temperature on due to the thermal expansion of the crystal and the fourth term allows for the effect of the interaction of electrons with the thermal vibrations of atoms (phonons). It is significant that irrespectively of the crystal structure the interaction of electrons with phonons always leads to a contraction of the width of the energy gap and to an increase of the electron conductivity. The value of the coefficient $\beta$ is $\beta=\beta_{o} / g^{2 / 3}(u)^{2}$ ( $\bar{u}$ is the sound velocity). The results of the calculations of the value $E_{g}(P, T)$ and the distribution of the intrinsic electron conductivity $\sigma-\sigma_{o} e^{-E_{g} / 2 K T}$ are given in table 1, our former results about the distribution of temperature in the Earth having been used.

We can see a satisfactory coincidence of the calculated values of $\sigma$ for the lower part of the mantle with the data of the value $\sigma$ according to McDonald (1957), obtained by means of geomagnetic variations. This indicates the plausibility of our initial assumption about the predominant role of the intrinsic electron conductivity in the lower layers of the mantle and confirms also that our former temperature calculations are in agreement with geomagnetic data.

Applying the above mentioned considerations we calculated that $\bar{\varepsilon}=500 \mathrm{~cm}^{-1}$. The curve of the olivine thermal conductivity against temperature can be best explained if to assume that besides lattice (phonon) thermal conductivity the radiative transfer and exciton thermal conductivity are present, the absorption increasing with $T$ the coefficient $\bar{\varepsilon}$ at the base of the mantle should increase up to the value and the length of the free run of excitons being $l=10^{-6} \mathrm{~cm}$ at $T=300^{\circ} K$ as it is the case for a number of other semi-conductors (Devyatkova 1957).

The theory of the lattice (phonon) part of the thermal conductivity of dielectrics in the range of high temperatures was dealt with in a number of papers. Most of them are based on the allowance for the anharmonicity of the thermal vibrations of the crystal lattice. The ultimate formulas somewhat differ from each other but it can be shown (Lawson 1957) that they are equivalent and are reduced to the unique expression:

$$
\lambda_{p h}=\hat{\lambda}_{o} / \tilde{\partial} \gamma^{2} \cdot T \cdot \chi^{3 / 2} \cdot \varrho^{1 / 2}
$$


where $\chi$ is the compressibility, $\gamma$ is the Grünaisen coefficient. Special place is occupied by the formula of Pomeranchuk (1943) who besides the anharmonicity took into account the dispersion of the thermal vibrations of the lattice. It can not be reduced to expression [4] and is written as follows:

$$
\lambda_{p h}=\lambda_{o} \varrho^{2 / 3} \frac{u^{3} \cdot u^{1 / 2}}{m^{5 / 4}}
$$

Since the temperature of the matter of the Earth's mantle is very high. close to the temperature of melting $T_{m}$ we can use the relations existing at such temperatures. Inserting the compressibility value from the Lindeman relation instead of $\chi$ into [4] we obtain the following relation. between $\lambda_{p h}$ and $T_{m}$ (Keyes R. W. 1959).

$$
\lambda T=B_{o} \cdot T_{m}^{3 / 2} \cdot \varrho^{2 / 3} / A^{7 / 6}
$$

where

$$
B_{o}=R^{3 / 2} / 3 \gamma^{2} \varepsilon^{2} \cdot N_{o}^{1 / 3}
$$

$A$ - is the average atom weight, $\varepsilon$ is the amplitude of the thermal vibrations at which melting begins, $N_{o}$ is the Avogadro number. The value $B_{o}$ can be considered constant to a rough approximation. The validity of this relation is tested by Keyes on the experimental material for more than thirty dielectric crystals. A similar transformation can be made with the Pomeranchuk formula and we can obtain:

$$
\lambda T^{5 / 4}=B_{1} \varrho^{2 / 3} T_{m}^{2} / A^{3}
$$

Inserting the values typical for the mantle of the Earth into formula [6] we shall find that the values in the mantle will be less than the curves of $T_{m}$ according to Uffen R. I. (1952) and Zharkov (1959) and taken by McDonald (1959). A better approximation to these curves is obtained if to use relation [7] (which testifies in favour of the Pomeranchuk formula [5]) and to increase the average atom weight of the material in the lower part of the mantle by adding of a percent of the ortosilicates of iron to olivine.

We must not overestimate the accuracy of these semi-empiric relations. But since our knowledge about the behaviour of the material 
in the deep interior of the Earth is very scant it is sometimes very important to establish that different values do not contradict each other. An example of it is the above given consideration as well as the comparison of the electro-conductivity pattern with the distribution of temperature.

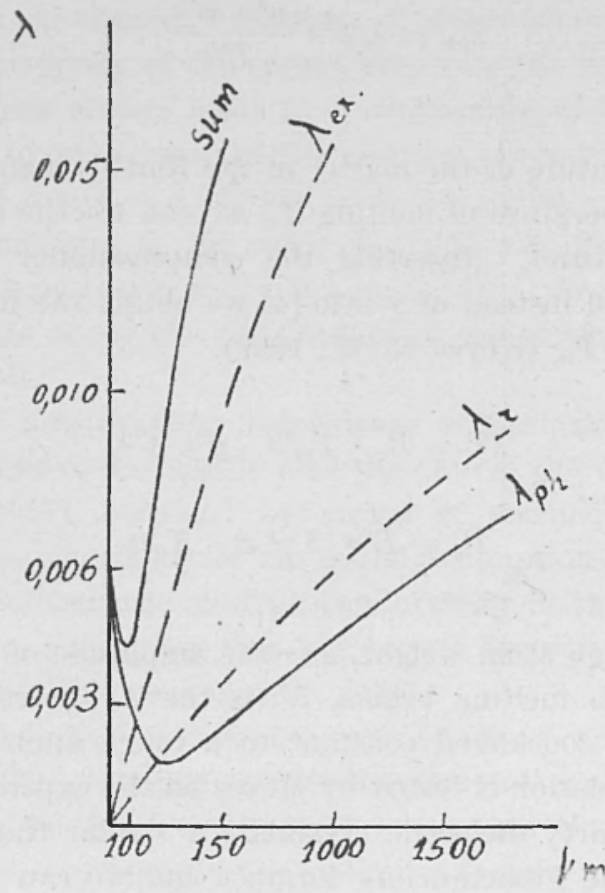

Fig. 2. - Variation of the total thermal conductivity and its components: lattice/phonon $/ \lambda_{p h}$, radiative $\lambda_{r}$ and exciton $\lambda_{e x}$ in the upper mantle.

A comparative distribution of the three components of the thermal conductivity in the Earth's mantle is represented in fig. 2. The temperature distribution for the present moment was taken from our paper (1958) for the calculation of the curves.

We see that the minimum of the thermal conductivity in the upper layers of the mantle becomes sharper than it was earlier without the allowance for the exciton component. The thermal conductivity at the base of the mantle exceeds its value at the surface by almost two orders. However this does not practically change the inferences about 
the process of the thermal history of the Earth made earlier without taking into account the exciton component on the assumption that the absorption coefficient of the radiative energy $\varepsilon=10 \mathrm{~cm}^{-1}$.

Let us consider now the case of the infinitely great thermal conductivity in the layers of the mantle lying below the level of the first hunrred kilometers. Our former calculations as well as the calculations

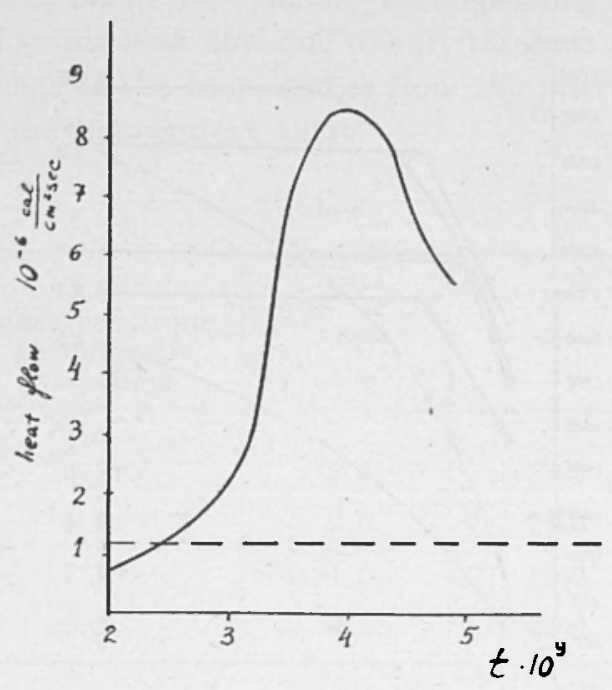

Fig. 3. - Variation of the heat flow with time at the infinitely great conductivity in the mantle.

made by McDonald (1959) showed that at the depth of 100 to $700 \mathrm{~km}$ the temperature was close to $T_{m}$ and in this region conditions seem to have been favorable for convection. The moment of the appearance of such a layer refers to the time of $3.10^{9}$ years ago. Together with Van Zin Di we calculated the temperature distribution and heat flow which can have existed in such an extreme case when the thermal conductivity is infinitely great in the indicated layer. The calculations were made by Lukvanov's hydrointegrator by the method described in our paper (1958). The infinitely great thermal conductivity was reached by a complete removing of the corresponding hydraulic resistances. Fig. 3 and 4 represent the results of the calculations of the heat flow and temperature distributions for different moments of time. We can-see that the Earth began cooling only $(1.0-1.5) \cdot 10^{8}$ years after the beginning 
of convection. However the heat flow at this period exceeds its modern observed value by 5 to 8 times. This indicates the unreality of the initial assumption about the existence of an intensive transfer of heat by convection in the mantle up till now and shows that such convection embracing a continuous belt in the Earth must have stopped by the present time. With the end of such convection the Earth's interior must have begun heating anew. This calculation can not be believed as an ulti-

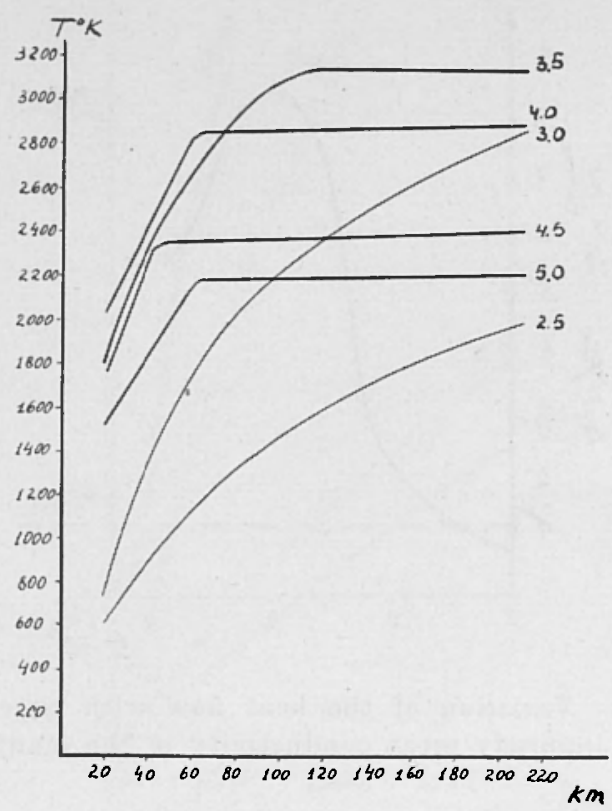

Fig. 4. - Variation of temperature with the depth for different moments of time at the infinitely great conductivity.

mate proof of the absence of convection in the Earth's interior. The convection could have occurred slower. It is essential that in this case according to our calculations a less intensive convection can not have led the globe to the state of great cooling.

We can not deny a convective transfer of heat in the Earth's mantle. It was rather of a local character in the form of the carrying out of heat when the sialic material was transferred from the mantle to the surface in the process of the crust formation. Let us consider such case. The amount of heat $Q$ carried out by the matter that formed the Earth's crust is estimated by us on the assumption that all the matter of the 
crust is transferred from depths where the temperature was by $\Delta T=$ $1000-3000^{\circ} \mathrm{C}$ greater than in the crust. Then $Q=V_{c} \cdot \varrho_{c} \cdot c_{c} \cdot \Delta T$, where $V_{c}$ is the crust volume, $o_{c}$ and $c_{c}$ are density and heat capacity of crustal rocks. For a period of time $\Delta t$ this makes a contribution to the surface heat flow equal to $\Delta q=\frac{Q}{s \cdot \Delta t}$ where $S-$ is the surface of the Earth. Thus for the $60 \mathrm{~km}$ crust as in geosynclinals at $\Delta t=3.10^{9}$ years the value $\Delta q=0,06$ or $0.2 \times 10^{-6} \mathrm{cal} / \mathrm{cm}^{2} \cdot \mathrm{sec}$ depending on the value $\Delta T$ should be added to the heat flow due to only the heat generation in the crust. The carriage of the heat sources from the interior should lay to the decrease of the temperature there.

Table 1

\begin{tabular}{|r|c|c|c|c|}
\hline $\mathrm{km}$ & $\begin{array}{c}\sigma \\
\text { observed from } \\
\text { geomagnetic } \\
\text { variations }\end{array}$ & $E_{g}$ & $T$ & $\begin{array}{c}\sigma \\
\text { theoretical }\end{array}$ \\
\cline { 2 - 4 } 750 & $2.10^{-2}$ & 2.6 & 2920 & $2.10^{-2}$ \\
1500 & $4.10^{-1}$ & 2.0 & 3960 & $2.10^{-1}$ \\
2000 & $7.10^{-1}$ & 1.5 & 4160 & $5.10^{-1}$ \\
2900 & 2.2 & 0.6 & 4300 & 2.1 \\
\hline
\end{tabular}

In reality the geological periods connected with the lifting of the material from the mantle corresponded to some intervals of time $<3.10^{9}$ years alternating with the states of relative rest.

If the process of the material lifting is concentrated for instance say at the interval of $\Delta t=1.10^{7}$ years then $\Delta q=(10-25) \cdot 10^{-6}$, if $\Delta T=1000$ to $3000^{\circ}$. This can explain the anomalously great value of the heat flow observed on young ridges in the Atlantic and Pacific ocean by Sir Edward Bullard and Von Herzen (1959).

If there were 2 or 3 such periods $\Delta t$ of the material carriage then $\Delta q$ must have been equal to $(5-12) \cdot 10^{-6}$ or $(3-8) \cdot 10^{-6}$ each time. Then the heat flow must have amounted to its maximum several times during the thermal history of the Earth.

The conclusion on the steady increase of the heat flow in the processes of the thermal history of the Earth, made by B. J. Levin and Majeva (1960) is schematic. They did not take into account that during a transference of sources to the surface, part of the heat is car- 
ried out also by the masses of the matter heated inside the hot interior, and that such process was not continual. The periods of the sialic material carriage alternated with the relative rest periods. Indeed geological data imply (Beloussov 1960) that the formation of the Earth's crust was not uniform in space and time.

At one and the same moment of time different areas of the Earth's (rust were at different stages of the development. The position of the heat flow maxima in time depends on the concrete history of a given reological region. In different areas of the Earth's crust the maximum of the heat flow could have been reached in different time. In the light of all that was said above it now seems more reasonable to put the problem about the thermal regime of locol areas of the Earth's crust instead of the regime of the crust as a wholl. This regime is different in different areas. Such consideration will depend completely on the geological history of the region, its past and present volcanic activity.

ACKNOWLeDGMENTS.

I wish to express my appreciation to Prof. B. I. Davjdov, Dr. S. P. Clark, Prof. R. Uffen and Prof. D. C. Tozer for helpful discussions.

\section{$S U M M A R Y$}

The efficiency of the lattice, radiative and exciton thermal conductivities in the Earth's mantle is considered. The mechanism of the variation of the activation energy for the electron intrinsic conductivity with temperature and pressure is considered.

The schematic calculation of the effect of the convective currents on the heat losses of the Earth is performed. Such effect should not stimulate a considerable cooling of the whole Earth now as the heat losses would then surpass the observal heat flow. Perhaps, the convection should cease by the present time.

The surface heat flow may have its several maximums in the past due to the heat carriage by moving of the sialic material from the hot interior to the crust in the corresponding geological periods.

\section{RIASSUNTO}

Si considera l'efficienza del reticolo, la conduttivita radioattiva $e$ termica dell' eccitone nella crosta terrestre. Si considera il meccanismo della variazione dell'energia di attivazione per la conduttivita intrinsica dell'elettrone rispetto alla temperatura e alla pressione. 
Si effettua il calcolo schematico dell'effetto delle correnti convettive sulle perdite di calore della Terra. Tale effetto non dovrebbe provocare un notevole raffreddamento di tutta la terra, poiche le perdite di calore supererebbero in tal modo il flusso di calore in osservazione. Forse la convezione dovrebbe cessare all' epoca attuale.

Il flusso di calore alla superfice pù̀ avere $i$ suoi vari massimi nel passato, dovuti al trasporto di calore provocato dal movimento del materiale sialico dall'interno caldo alla crosta, nei corrispondenti periodi geologici.

\section{REFERENCES}

(1) Balchan A. S., Drickamer H. G., Effect of Pressure on the Spectra of Olivine and Garnet. "J. App. Phys. ", 30, 9, (1959).

$\left(^{2}\right)$ CLARK S. P., Effect of Radiative Transfer on Temperatures in the Earth. "Bull. Geol. Soc. Am. ", 67, 1123, (1956).

$\left.{ }^{3}\right)$ - Absorption Spectra of Some Silicates in the Visible and Near Infrared, "Am., Mineral ", 42, (1957).

(4) Devyatkova E. D., жT中, 27, 3, (1957).

$\left(^{5}\right)$ Zharkov V. N., Melting Points of the Earth's Mantle and Iron ai High Pressures. "Izvestiya AN SSSR ", ser. geophys., 1, (1959).

(6) Haken H., Die Theorie des Exzitonts in festen Forper, Fortschritte der Physik. 6. 271-334, (1958).

(7) Jamieson I. C., Lawson A. W., Heat Transfer in the Earth. "J. Geol. ", $11,5,(1958)$.

(8) Keyes R. W., High Temperature Thermal Conductivity of Insulating Crystals: Relationship to the Melting Point. "Phys. Rev.", 115, 3, 564, (1959).

$\left.{ }^{9}\right)$ KINGERY, The Variation of Conductivity with Temperature for Some Silicates. "J. Am. Cer. Soc. ", 37, 2, (1954).

(10) I.Awson A. W., On the High Temperature Heat Conductivity of Insulators. "J. Phys. Chem. Sol. ", 3, 1/2, (1957).

(11) Lunimova H. A., Thermal History of the Earth with Consideration of the Variable Thermal Conductivity. "Geoph. J. Roy. Astr. Soc.", 1, 2, (1958).

${ }^{\left({ }^{12}\right)}$ - On Temperature Gradients in Earth's Opper Layers and Possibility of Explanation of Low Velocities. "Izvestiya AN SSSR ", ser. geoplyss., 12, (1959).

(13) MoDonatd G. I., Calculations on the Thermal History of the Earth. "J. Geoph. Res. ", 64, 11, (1959).

(14) Martienssen W., The Optical Absorption Edge in Ionic Crystals. "J. Phys. Chem. Soc. ", 8, 264, (1959). 
( $\left.{ }^{15}\right)$ McDonald K. L., Penetration of the Geomagnetic Secular Field through a Mantle with Variable Conductivity. "J. Geophys. Ros. ", 62, 1, (1957).

(16) Pomeranciun J., Heat Conductivity of Dielectrics at High Temperatures. "J. Phys. USSR », 7, 5, (1943).

(17) Preston F. W., Thermal Conductivity in the Depth of the Earth. "Am. J. Sci. ", 254, 754, (1956).

(18) Reiling G. H., Henslex E. B., Fundamental Optical Absorption in Magnesium Oxide. "Phys. Rev.". 112, 4, 1106, (1958).

(19) Tozer D. C., The Electrical Properties of the Earth's Interior. "Physics and Chemistry of the Earth ", 3, Perg. Press L.N.L., 1959.

${ }^{(20)}$ Uffen R. Y., A Method of Estimating the Melting Point Gradient in the Earth's Mantle "Trans. Am. Geophys. Un.", 33, 6, (1952).

(21) BeLoussov V. V., The development of the Earth and Tectogenesis. "Soviet Geologie ", 7, (1960).

${ }^{(22)}$ Levin B. J., Majeva S. V., On the Thermal History of the Earth. "Bull. Acad. Sci. USSR ", geophys. ser. III -61, 2, (1960). 